\title{
EDITORIAL
}

\section{What's new in ARDS: can we prevent it?}

\author{
Roy G. Brower ${ }^{1}$ and Massimo Antonelli $i^{*}$ (I)
}

๑) 2016 Springer-Verlag Berlin Heidelberg and ESICM

\section{Rationale for prevention}

The incidence of acute respiratory distress syndrome (ARDS) is variable, depending on definitions, sampling techniques, and geographic region, but it is almost always a morbid and frequently fatal disorder. Survivors frequently suffer from debilitating sequelae [1]. Most clinical trials of promising treatments for ARDS failed to demonstrate beneficial effects.

One possible reason for these disappointing trials is that the experimental therapies were administered after the lungs had been affected by severe inflammatory processes. These therapies may be more effective if they are administered earlier in patients' conditions such as severe sepsis or trauma, before onset of ARDS. An example of a successful prevention approach is the use of lower tidal volumes in patients who did not have ARDS when mechanical ventilation was initiated. This was associated with a decrease in the frequency of hospital-acquired ARDS [2].

\section{Challenges to prevention strategies Identification of at-risk patients}

The first step to prevention of ARDS is to identify patients most likely to benefit from the prophylactic interventions. Several recent prevention studies selected patients undergoing surgical procedures in which there was substantial risk of postoperative ARDS, such as cardiac or esophageal surgeries [3, 4]. In these studies, the preventive interventions were delivered perioperatively, before onset of ARDS.

The lung injury prediction score (LIPS) [5] is a promising tool for risk stratification at hospital admission of patients without ARDS. To develop this prediction

\footnotetext{
*Correspondence: massimo.antonelli@policlinicogemelli.it

2 Department of Anesthesiology and Intensive Care Medicine, A. Gemelli University Hospital, Catholic University of Rome, Largo A. Gemelli, 8, 00168 Rome, Italy

Full author information is available at the end of the article
}

model, 5584 patients with sepsis, shock, pancreatitis, pneumonia, aspiration, high-risk trauma, and high-risk surgery were evaluated after hospital admission. The model included the identification of the risk modifiers (alcohol abuse, obesity, chemotherapy, hypoalbuminemia, $\mathrm{RR}>30, \mathrm{SpO}_{2}<95 \%, \mathrm{FiO}_{2}>35 \%, \mathrm{pH}<7.35$, and diabetes mellitus) that allowed a better stratification of the at-risk population. A LIPS $\geq 4$ points had a sensitivity of $69 \%$ and specificity of $78 \%$ for identifying patients who would develop ARDS after admission. The validity of LIPS for identifying patients at high risk for ARDS in the emergency department was confirmed in a recent external cohort [6]. The LIPS was used in the recently concluded Lung Injury Prevention Study-Aspirin (LIPS-A) [7].

Another tool for identifying patients at risk for ARDS is the early acute lung injury score (EALI) [8]. An EALI score $\geq 2$ had a sensitivity of $89 \%$, specificity of $75 \%$, and positive predictive value of $53 \%$. However, the EALI score has not been validated in an external cohort or used in a clinical trial for ARDS prevention.

A reliable biomarker-driven approach could be of great value to identify high-risk patients or to identify patients most likely to benefit from a new intervention. The combination of plasma angiopoietin-2 with LIPS was a better predictor for subsequent development of ARDS than either LIPS or angiopoietin-2 alone [6].

\section{Timing of preventive interventions}

The timing of preventive interventions for ARDS depends largely on whether the intervention is intended as primary, secondary, or tertiary prevention. Primary prevention aims to prevent disease or injury before it occurs. For ARDS this is only feasible in patients in whom the acute injury predisposing to ARDS can be predicted, such as those undergoing elective high-risk surgery or patients who need multiple transfusions $[9,10]$.

Secondary prevention aims to reduce the impact of a disease or injury, such as sepsis or aspiration, that has already occurred. The period between a patient's hospital

\section{Springer}


admission and when ARDS develops often occurs in the emergency department or on the in-patient ward. Many hospitalized patients with sepsis and other risk factors for ARDS are managed outside of the ICU [11]. Thus, screening for ARDS prevention trials must occur outside of the ICU.

Tertiary prevention aims to soften the impact of an ongoing illness or injury that may have long-lasting effects. Tertiary prevention studies in ARDS can also focus on reducing the complications and loss of function in survivors. At 1 year after hospital discharge, up to 70 and $36 \%$ of ARDS survivors suffer from cognitive and functional impairments, respectively [2]. Therefore, clinical trials aimed at improving these long-term outcomes of ARDS survivors are important.

\section{Trial design}

Effectiveness study designs may be appropriate to ensure safety and generalizability of prevention trial results. However, the risk-benefit ratios of preventive interventions are difficult to predict. Therefore, new clinical trial designs such as adaptive design may be used, although the implementation of these studies can be quite complex $[12,13]$.

When the incidence of ARDS is low and a new intervention entails minimal risks, pragmatic trial designs may be possible. These trials have broad inclusion criteria, few exclusion criteria, and the intervention is delivered in the usual care setting [14]. These trials usually enroll large populations to gain adequate power, compensating for the lower incidence of the outcome and the more heterogeneous population included in the study.

Cluster randomization is another alternative for critical care prevention trials design [15]. Instead of randomizing patients individually to an intervention, all patients within a cluster of ICUs or hospitals are randomized to an intervention or control group.

Many ARDS treatment trials utilized the composite clinical outcome of ventilator-free days (VFDs), a continuous outcome that combines mortality with time to successful weaning [16]. A primary outcome variable of VFDs allows enrollment of a smaller sample size than mortality, but there is the potential that a new treatment increases VFD and also mortality. These results would be uninterpretable.

\section{Ideas for prevention of ARDS}

Table 1 lists a selection of proposed approaches for secondary prevention of ARDS. Some of the proposed therapies are similar to those that were not effective in clinical trials in established ARDS. Others are newer, more novel approaches that have not been tested previously in severe inflammatory diseases.

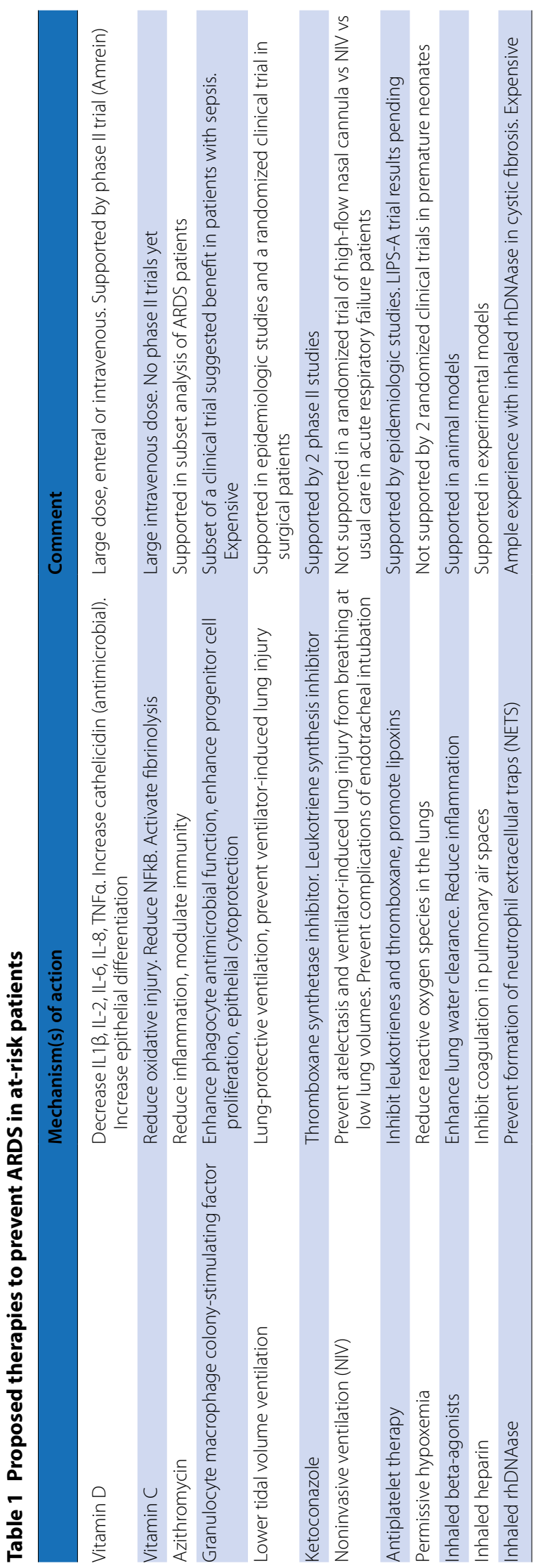




\section{Summary}

There is increasing emphasis on early identification of patients at risk for ARDS and implementing strategies to prevent ARDS. There are major challenges in selecting appropriate at-risk patients, timing of interventions, study design, and outcome measures. With the recent increase in trials of preventive strategies, these challenges might be met and efficacious treatments identified.

The focus on prevention is represented by the new US National Institutes of Health (NIH) Prevention and Early Treatment of Acute Lung Injury (PETAL) Network. This network, which succeeded the ARDS Network, is designed to conduct clinical trials of promising treatments for ARDS prevention.

\section{Author details}

${ }^{1}$ Pulmonary and Critical Care Medicine, Johns Hopkins University School of Medicine, Baltimore, USA. ${ }^{2}$ Department of Anesthesiology and Intensive Care Medicine, A. Gemelli University Hospital, Catholic University of Rome, Largo A. Gemelli, 8, 00168 Rome, Italy.

Received: 25 January 2016 Accepted: 16 February 2016

Published online: 1 March 2016

\section{References}

1. Herridge MS, Tansey CM, Matte A et al (2011) Functional disability 5 years after acute respiratory distress syndrome. N Engl J Med 364:1293-1304

2. Li G, Malinchoc M, Cartin-Ceba R, Venkata CV, Kor DJ, Peters SG, Hubmayr RD, Gajic O (2011) Eight-year trend of acute respiratory distress syndrome: a population-based study in Olmsted County, Minnesota. Am J Respir Crit Care Med 183(1):59-66

3. Kim JC, Shim JK, Lee S et al (2012) Effect of combined remote ischemic pre-conditioning and postconditioning on pulmonary function in valvular heart surgery. Chest 142:467-475
4. Li C, Xu M, Wu Y et al (2014) Limb remote ischemic preconditioning attenuates lung injury after pulmonary resection under propofolremifentanil anesthesia: a randomized controlled study. Anesthesiology 121:249-259

5. Gajic O, Dabbagh O, Park P, Adesanya A, Chang S, Hou P et al (2011) Early identification of patients at risk of acute lung injury: evaluation of lung injury prediction score in a multicenter cohort study. Am J Resp Crit Care Med 183:462-470

6. Agrawal A, Matthay MA, Kangelaris KN et al (2013) Plasma angiopoietin-2 predicts the onset of acute lung injury in critically ill patients. Am J Respir Crit Care Med 187:736-742

7. Kor DJ, Talmor DS, Banner-Goodspeed VM et al (2012) Lung Injury Prevention with Aspirin (LIPS-A): a protocol for a multicentre randomised clinical trial in medical patients at high risk of acute lung injury. BMJ Open 2:e001606

8. Levitt JE, Calfee CS, Goldstein BA et al (2013) Early acute lung injury: criteria for identifying lung injury prior to the need for positive pressure ventilation. Crit Care Med 41:1929-1937

9. Shyamsundar M, McAuley DF, Shields MO et al (2014) Effect of simvastatin on physiological and biological outcomes in patients undergoing esophagectomy: a randomized placebo-controlled trial. Ann Surg 259:26-31

10. Hajjar LA, Vincent JL, Galas FR et al (2010) Transfusion requirements after cardiac surgery: the TRACS randomized controlled trial. JAMA 304:1559-1567

11. Rohde JM, Odden AJ, Bonham C et al (2013) The epidemiology of acute organ system dysfunction from severe sepsis outside of the intensive care unit. J Hosp Med 8:243-247

12. Chow SC (2014) Adaptive clinical trial design. Ann Rev Med 65:405-415

13. Patsopoulos NA (2011) A pragmatic view on pragmatic trials. Dialogues Clin Neurosci 13:217-224

14. Treweek S, Zwarenstein M (2009) Making trials matter: pragmatic and explanatory trials and the problem of applicability. Trials 10:37

15. Christie J, O'Halloran P, Stevenson M (2009) Planning a cluster randomized controlled trial: methodological issues. Nurs Res 58:128-134

16. Schoenfeld DA, Bernard GR, Network A (2002) Statistical evaluation of ventilator-free days as an efficacy measure in clinical trials of treatments for acute respiratory distress syndrome. Crit Care Med 30:1772-1777 